University of Wollongong

Research Online

Faculty of Engineering and Information

Faculty of Engineering and Information

Sciences - Papers: Part B

Sciences

2017

Improvement of the Sign Determination Method for Data-Driven respiratory signal in TOF-PET

Ottavia Bertolli

University College London

Simon R. Arridge

University College London

Charles Stearns

Ge Healthcare

Scott Wollenweber

Ge Healthcare

Brian F. Hutton

University of Wollongong, b.hutton@ucl.ac.uk

See next page for additional authors

Follow this and additional works at: https://ro.uow.edu.au/eispapers1

Part of the Engineering Commons, and the Science and Technology Studies Commons

Research Online is the open access institutional repository for the University of Wollongong. For further information contact the UOW Library: research-pubs@uow.edu.au 


\title{
Improvement of the Sign Determination Method for Data-Driven respiratory signal in TOF-PET
}

\author{
Abstract \\ Respiratory gating and motion correction can increase resolution in PET chest imaging, but require a \\ respiratory signal. Data-Driven (DD) methods aim to produce a respiratory signal from PET data, avoiding \\ the use of external devices. Principal Component Analysis (PCA) is an easy to implement DD algorithm \\ whose signals, however, are determined up to an arbitrary factor. The direction of the motion represented \\ by its signal has to be determined. In this work we present the extension to TOF data of a previously \\ presented sign-determination method. Furthermore, we propose the application of a selection process in \\ sinogram space, to automatically select the areas of the data mostly affected by respiratory motion. The \\ performance of the updated signdetermination method is evaluated on patient data, and the effect of TOF \\ information and masking process is investigated also in terms of quality of the PCA respiratory signal.

\section{Disciplines \\ Engineering | Science and Technology Studies}

\section{Publication Details} \\ Bertolli, O., Arridge, S., Stearns, C. W., Wollenweber, S. D., Hutton, B. F. \& Thielemans, K. (2017). \\ Improvement of the Sign Determination Method for Data-Driven respiratory signal in TOF-PET. 2017 IEEE \\ Nuclear Science Symposium and Medical Imaging Conference, NSS/MIC 2017 - Conference Proceedings \\ (pp. 1837-1840). United States: IEEE.
}

\section{Authors}

Ottavia Bertolli, Simon R. Arridge, Charles Stearns, Scott Wollenweber, Brian F. Hutton, and Kris Thielemans 


\title{
Improvement of the Sign Determination Method for Data-Driven respiratory signal in TOF-PET
}

\author{
Ottavia Bertolli, Student Member, IEEE, Simon Arridge, Charles W Stearns, Fellow, IEEE, \\ Scott D Wollenweber, Senior Member, IEEE, Brian F Hutton, Senior Member, IEEE, \\ Kris Thielemans, Senior Member, IEEE
}

\begin{abstract}
Respiratory gating and motion correction can increase resolution in PET chest imaging, but require a respiratory signal. Data-Driven (DD) methods aim to produce a respiratory signal from PET data, avoiding the use of external devices. Principal Component Analysis (PCA) is an easy to implement DD algorithm whose signals, however, are determined up to an arbitrary factor. The direction of the motion represented by its signal has to be determined. In this work we present the extension to TOF data of a previously presented sign-determination method. Furthermore, we propose the application of a selection process in sinogram space, to automatically select the areas of the data mostly affected by respiratory motion. The performance of the updated signdetermination method is evaluated on patient data, and the effect of TOF information and masking process is investigated also in terms of quality of the PCA respiratory signal.
\end{abstract}

Index Terms-PET, PCA, respiratory motion, sign determination

\section{INTRODUCTION}

$\mathbf{P}$ ATIENT respiratory motion is one of the major causes of degradation of PET images when imaging the chest. In order to reduce this effect, respiratory gating and subsequent motion correction are advisable, therefore a respiratory signal is needed. In clinical practice this is obtained using external devices that detect the respiratory motion, whereas it has been shown that Data-Driven methods can produce a reliable respiratory signal while only requiring the use of the raw data [1]. Among the Data-Driven methods available, Principal Component Analysis (PCA) is an easy to implement algorithm that produces reliable respiratory signals [2], however its signals are determined up to an arbitrary factor. The direction of the motion represented by the PCA signal has to be determined in order to perform motion correction correctly. In previous work, we have developed a method to determine the direction of the PCA signal for non-TOF PET data [3]. However, TOF provides increased spatial information [4] that benefits the PCA method

This work was supported by EPSRC Industrial CASE studentship (13220093), co-sponsored by GE Healthcare, and by the National Institute for Health Research, University College London Hospitals Biomedical Research Centre.

O. Bertolli, K. Thielemans and B. F. Hutton are with the Institute of Nuclear Medicine, UCLH, 235 Euston Road (T-5), London, NW1 2BU, UK email: ottavia.bertolli.13@ucl.ac.uk

S. Arridge is with the Centre for Medical Image Computing, University College London, Gower Street, London, WC1E 6BT, UK

B. Hutton is also with the Centre for Medical Radiation Physics, University of Wollongong, Australia

C. W. Stearns and S. D. Wollenweber are with GE Healthcare, Waukesha, WI, USA.

Manuscript received November 17, 2017.
[5], therefore in this work we extend the sign determination method to TOF data. We show that the method's performance improves with the use of TOF sinograms, together with the selection of the central part of the sinogram data. Additionally, the quality of the PCA respiratory signal is also evaluated.

\section{Methods}

We briefly describe the PCA method on non-TOF data (see [2] for details), that will later be extended to the TOF case. PCA is applied to listmode data unlisted into sinograms with time resolution of $500 \mathrm{~ms}$, that are spatially downsampled to decrease the noise. The final sinogram is defined as $d(r, z, \phi, t)$. The respiratory PC is the one whose weight factor $w(t)$ has the highest peak in the respiratory frequency band (as in [2]) and is representative of the respiratory-induced changes in the data.

The assumption at the base of the sign-determination method in [3] is that this motion is mostly in the axial direction. In [3] a factor is created from the raw data, simulating the effect of rigid motion in the axial direction, that attempts at recreating the features of the respiratory PC and is related to a motion in a known direction (i.e. where maxima in the displacement correspond to the end-inhalation state). This factor is referred to as WeightedGradSino and it is in sinogram space. To generate its temporal signal (equivalent to the weight factor for the respiratory PC) the inner-product between the WeightedGradSino and the dynamic sinogram $d(r, z, \phi, t)$ is evaluated:

$$
u(t)=\sum_{r, z, \phi} \text { WeightedGradSino }(r, z, \phi) d(r, z, \phi, t)
$$

This signal is generated so as to have an established relationship with the true patient motion (present in the data), therefore comparing it with the PC signal $u(t)$ will determine whether the latter needs to be corrected (by flipping its sign). This comparison is performed via evaluation of the Pearson correlation between $u(t)$ and $w(t)$ :

$$
\operatorname{corr}(w, u)=\frac{\sum_{t}(w(t)-\bar{w})(u(t)-\bar{u})}{\sqrt{\sum_{t}(w(t)-\bar{w})^{2}(u(t)-\bar{u})^{2}}}
$$

when this value is found negative the sign of the PC signal needs to be corrected. This method will be referred to as CorrWeights, as it is the correlation between the weight of the PC and of that of the WeightedGradSino.

When TOF-PET data is available, the raw data can be unlisted into 5-dimensional TOF sinograms, with the added dimension 
given by the TOF bin: $d(r, z, \phi, \tau, t)$. The PCA method and the sign-determination method can be easily extended to be applied to these 5-dimensional sinograms. In our previous work, we have shown that applying PCA on the parts of the TOF sinogram more likely to be affected by respiratory motion increases the quality of the PCA signal by avoiding non-respiratory changes presented in the data [5] (e.g. bulk motion, arm motion). Here we investigate the effect on the sign determination when specifically targeting the area of the TOF sinograms where the assumption that variation in the data is due to axial motion is likely to be correct.

The selection of the data to be given as input to PCA (for the respiratory signal extraction) and for the creation of the WeightedGradSino and its signal $u(t)$, was performed by creating a cylinder (diameter $20 \mathrm{~cm}$ ) in image space with value 1 and 0 outside, placed at the center of the FOV, and then applying non-TOF and TOF forward-projection to obtain the areas in the sinogram that are comprised within its volume. A binary mask was created assigning a value of 1 to all the non-zero elements in the sinogram and 0 elsewhere. The non-TOF and TOF sinogram data were then masked with the corresponding cylinder sinogram and the result was used as input to PCA and to the sign-determination algorithm.

The correlation between $w(t)$ and $u(t)$, that is the decision metric for correcting the sign of the PCA generated signal, was evaluated with and without the pre-selection of the data in the non-TOF and TOF case. The Pearson correlation of the PC respiratory signal with the external device signal was also evaluated to investigate whether using selected data increases the quality of the PCA output.

The analysis was performed on 28 FDG oncology patient datasets using activity levels according to routine clinical protocols (16 patients acquired on Discovery 690 PET/CT and 12 patients acquired on Discovery 710 PET/CT). The duration of the acquisitions ranges from 180s to 300s. The acquisitions were monitored by the Varian ${ }^{\circledR}$ Real-time Position Management ${ }^{\mathrm{TM}}$ (RPM) device, and its signal was utilised to check on the direction of the PCA signal after sign correction. The TOF resolution of the scanners is 550ps [6], and data are acquired in 55 TOF bins, of $89 \mathrm{ps}$ width. The listmode files were unlisted to a time resolution of $500 \mathrm{~ms}$ in non-TOF sinograms, and also in TOF-sinograms with a total number of TOF bins of 5 and 11 (where 11 and 5 TOF bins were summed together, respectively).

For each patient the sinograms were also subdivided in independent shorter time intervals of $25 \mathrm{~s}, 50 \mathrm{~s}, 100 \mathrm{~s}, 200 \mathrm{~s}$ and 300 s (for the longest studies) in order to assess the performance of PCA and the sign-determination method on smaller amount of data. To evaluate the performance with fewer counts, data was also unlisted into sinograms with random rejection of counts, keeping a percentage of the total counts equal to $3 \%, 6 \%$, $12.5 \%, 25 \%$ and $50 \%$. For this sub-study, the total duration of the sinogram was chosen as 180s (duration of the shortest acquisitions).

\section{RESULTS}

In Fig. 1 the coronal view of one patient sinogram is shown after multiplication with the cylindrical mask. It can be seen

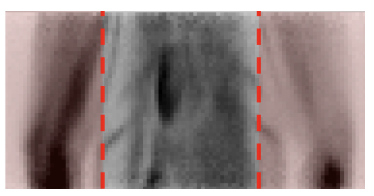

(a)

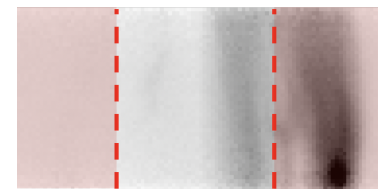

(b)
Fig. 1: (a) Example of the coronal view of the TOF projections for the $3^{\text {rd }}$ TOF bin, that corresponds to the central area. (b) $2^{\text {nd }}$ bin, that corresponds to areas further away from the center of the FOV. The shaded area is the part that is masked away when using the cylindrical mask.

that the result of the selection in sinogram space corresponds to the central part of the patient (the darker areas in the sinogram are the lungs), therefore disregarding the outer areas where no respiratory motion is expected to occur. Note that in the TOF case the proposed masking process also selects different TOF bins, therefore effectively removing parts of the TOF projection data that include counts generated outside of the $20 \mathrm{~cm}$ cylinder volume.

\section{A. Analysis with respect to shorter intervals}

Figure 2 shows the mean value of the correlation of the PCA respiratory signal with the RPM, averaged over all patients, for all the different interval durations taken into account. Figure 3 shows the correlation with the RPM as boxplots for the two most extreme case: shortest and longest duration (25s and 300s).

Figure 4 and 5 show the mean of the CorrWeights value averaged over all patients for all interval durations, and then the boxplot for the $25 \mathrm{~s}$ and $300 \mathrm{~s}$ cases. Table I shows the failure rate of the method in determining the correct direction of motion, evaluated on all intervals taken into account (583 in total).

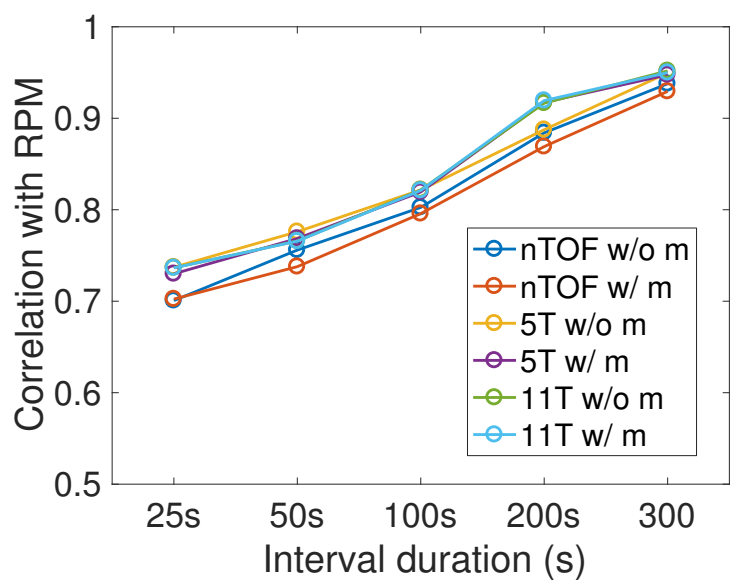

Fig. 2: The mean values for each interval duration are obtained averaging the correlation with the RPM of the signals obtained for all patients. This process is applied for all the various data configurations: non -TOF sinograms without and with masking (respectively $n T O F, w / o m$ and $n T O F w / m$ ); TOF sinograms with 5 and 11 TOF bins without and with masking (respectively 5TOF $w / O \mathrm{~m}$ and $\mathrm{w} / \mathrm{m}$ and IITOF $w / o \mathrm{~m}$ and $\mathrm{w} / \mathrm{m}$ ). 

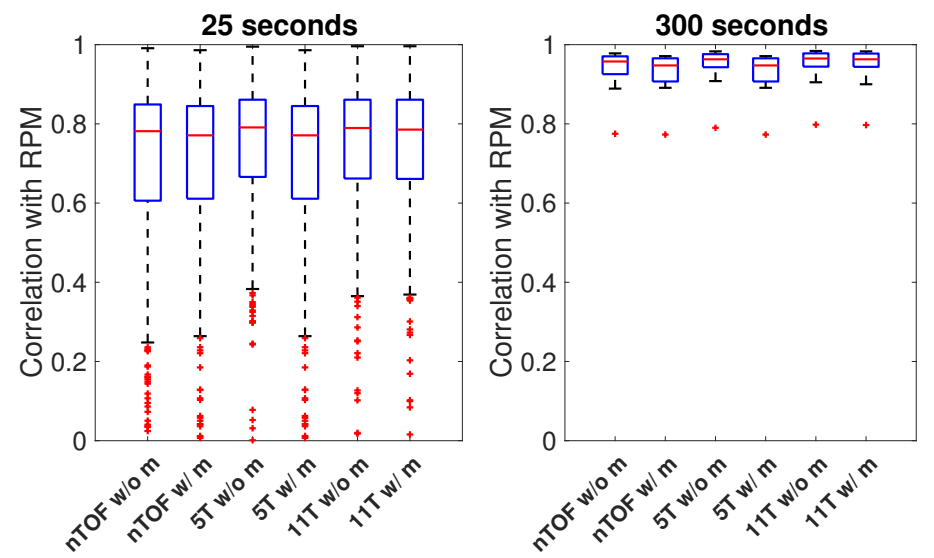

Fig. 3: Boxplot of the correlation with the RPM for the two most extreme cases: the shortest intervals $(25 \mathrm{~s}, 322$ total intervals) and the longest ones (300s, 16 total intervals).

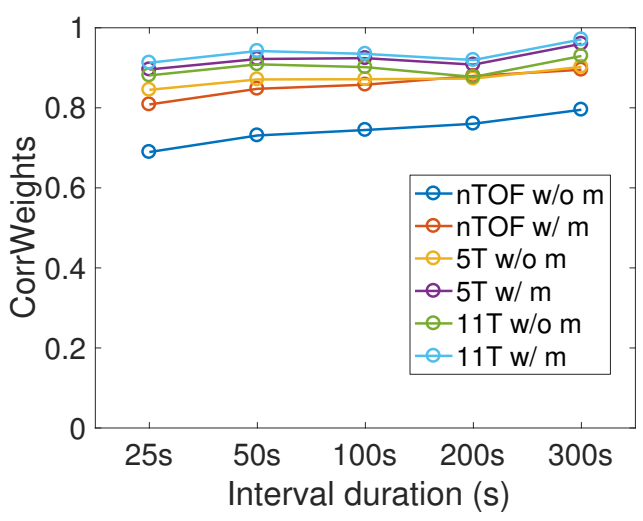

Fig. 4: Mean of the CorrWeights value (see Eq. 2) for all the evaluated intervals for all the various data configurations (as in Fig.2) .

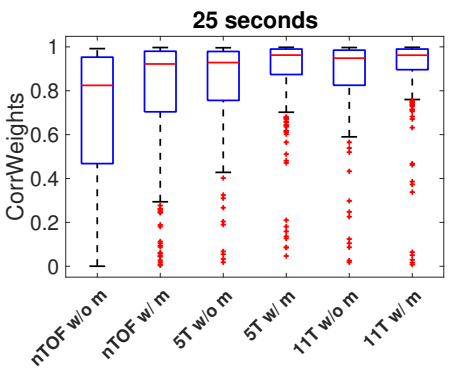

(a)

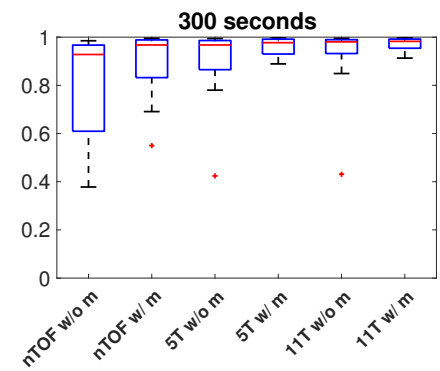

(b)
Fig. 5: Boxplots of the CorrWeights values with respect to the interval duration for all the various data configurations, for the $25 \mathrm{~s}$ and $300 \mathrm{~s}$ intervals.

\section{B. Analysis with respect to fewer counts}

Figure 6 shows the mean value of the correlation of the PCA respiratory signal with the RPM, averaged over all patients, for all the different percentages of utilised counts. Similarly, Figure 7 shows the mean of the CorrWeights values, and Figure 8 the spread of the values for all data configurations with $12.5 \%$ and $100 \%$ of the data. Table I shows the failure rates of the
TABLE I: Failure rate of the method in determining the correct direction of motion, on all intervals taken into account (with duration equal to $25 \mathrm{~s}, 50 \mathrm{~s}, 100 \mathrm{~s}, 200 \mathrm{~s}$ and $300 \mathrm{~s}$, for a total of 583 intervals).

\begin{tabular}{cccccc}
\hline $\begin{array}{c}\text { non-TOF } \\
\text { w/o m }\end{array}$ & $\begin{array}{c}\text { non-TOF } \\
\text { w/ m }\end{array}$ & $\begin{array}{c}5 \text { TOF } \\
\text { w/o m }\end{array}$ & $\begin{array}{c}5 \text { TOF } \\
\text { w/ m }\end{array}$ & $\begin{array}{c}11 \text { TOF } \\
\text { w/o m }\end{array}$ & $\begin{array}{c}11 \text { TOF } \\
\text { w/ m }\end{array}$ \\
\hline 2.7 & 1.0 & 0.7 & 0.5 & 0.9 & 0.9 \\
\hline
\end{tabular}

sign-determination method for all data configurations and for all count levels.

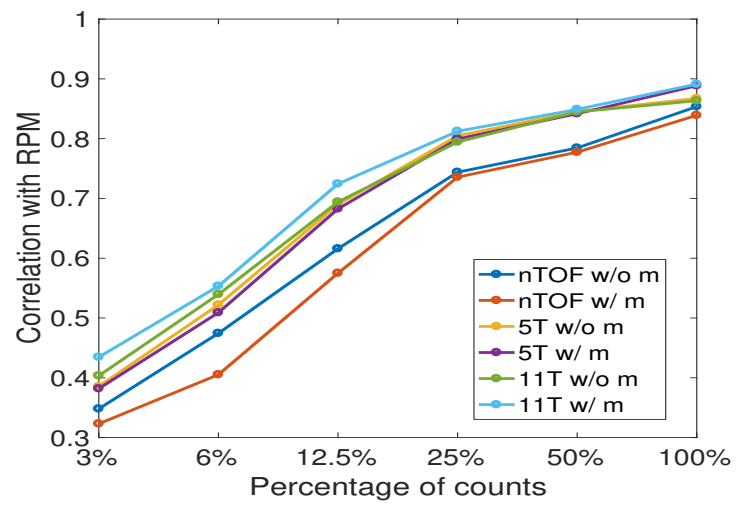

Fig. 6: Mean value of the correlation of the PCA signal with the RPM, averaged over all patients, for all count levels.

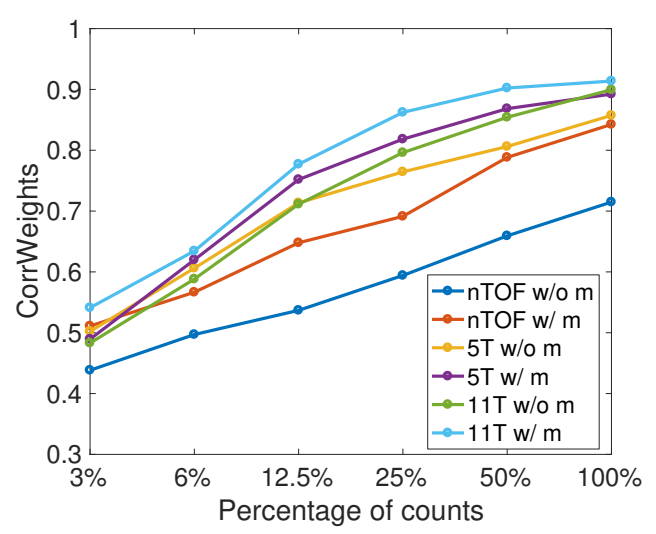

Fig. 7: Mean of the output values of CorrWeights, averaged over all patients, for all count levels.

TABLE II: Failure rate of CorrWeights in determining the correct direction of motion, on all the 28 patients, for the all the various percentages of utilised counts.

\begin{tabular}{l|cccccc}
\hline$\%$ & $\begin{array}{c}\text { non-TOF } \\
\text { w/o m }\end{array}$ & $\begin{array}{c}\text { non-TOF } \\
\text { w/ m }\end{array}$ & $\begin{array}{c}5 \text { TOF } \\
\text { w/o m }\end{array}$ & $\begin{array}{c}5 \text { TOF } \\
\text { w/ m }\end{array}$ & $\begin{array}{c}\text { 11TOF } \\
\text { w/o m }\end{array}$ & $\begin{array}{c}\text { 11TOF } \\
\text { w/ m }\end{array}$ \\
\hline 3 & 0.14 & 0.10 & 0.07 & 0.04 & 0.04 & 0 \\
6 & 0.04 & 0.2 & 0.07 & 0.04 & 0.04 & 0 \\
12.5 & 0.18 & 0.07 & 0.04 & 0 & 0 & 0 \\
25 & 0.21 & 0.07 & 0.07 & 0 & 0 & 0 \\
50 & 0.07 & 0.07 & 0.04 & 0 & 0 & 0 \\
100 & 0 & 0 & 0 & 0 & 0 & 0 \\
\hline
\end{tabular}




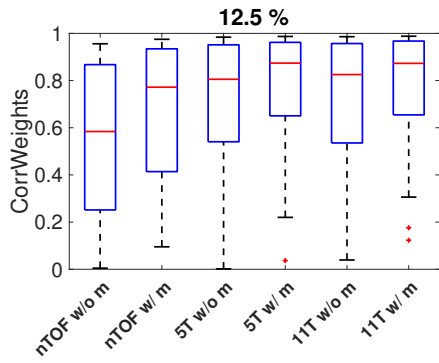

(a)

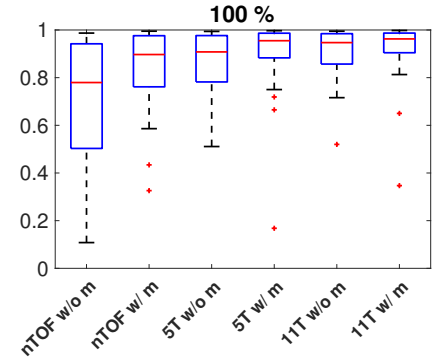

(b)
Fig. 8: Boxplots of the CorrWeights values for all the various data configurations, for the $12.5 \%$ and $100 \%$ of the counts.

\section{DISCUSSION}

The quality of the PCA respiratory trace and the performance of the sign-determination method have been evaluated, both on shorter time intervals and datasets with varying amount of counts. When using shorter time intervals, the use of the TOF sinograms (5 or 11 TOF bins) and of the cylindrical selection only had a small impact on the overall quality of the respiratory signal produced by PCA, as the mean values of the correlation with the RPM are slightly higher with TOF sinograms and use of the cylindrical masking, but the spread in the values is comparable to the non-TOF case without masking (see Figures 2 and 3). However, with regards to the sign-determination process, the exploitation of TOF information has proven to be beneficial, as the CorrWeights method yields higher values than in the nonTOF case and the failure rate decreases (see Figures 5 and 4 and Table I).

Moreover, the further application of a cylindrical mask in sinogram space, to select the central part of the data, has resulted in even higher CorrWeights values and therefore in a more robust method to correct the sign of the PCA signal.

When applying the methods on datasets with fewer counts, the use of TOF sinograms and masking improves both the quality of the respiratory signal (evaluated as correlation with the RPM, see Figure 2) and the performance of CorrWeights (see Figures 7 and 8). In particular, the failure rate of CorrWeights is null when utilising 11 TOF bins sinograms with the application of the cylindrical mask, as shown in Table II.

\section{CONCLUSION}

The extension of the CorrWeights sign-determination method to TOF, together with the application a cylindrical mask in sinogram space, has proven to be beneficial, as the method's output value increases and the failure rate decreases. The method has shown an improved performance both on short time intervals and datasets with fewer counts. Furthermore, the PCA respiratory signal has shown increased similarity to the RPM when using fewer counts if applied on TOF sinograms with cylindrical masking. No noticeable difference has been found in the case of shorter time intervals. In conclusion, when TOF-PET data is available and PCA Data-Driven respiratory detection is performed, it is advisable to apply both PCA and the described sign-determination method on TOF sinograms together with the application of a cylindrical mask, in particular for low count studies. The use of more TOF bins is beneficial.

\section{ACKNOWLEDGMENT}

We wish to thank François Bernard (BCCA, Vancouver, CA), Daniel McGowan (Oxford University Hospitals NHS Foundation Trust) and Simon Wan, Tom Sanderson and Raymond Endozo (Institute of Nuclear Medicine, UCLH, London) for the anonymized data.

\section{REFERENCES}

[1] A. L. Kesner, P. J. Schleyer, F. Büther, M. A. Walter, K. P. Schäfers, and P. J. Koo, "On transcending the impasse of respiratory motion correction applications in routine clinical imaging-a consideration of a fully automated data driven motion control framework," EJNMMI Physics, vol. 1, no. 1, p. 8, 2014.

[2] K. Thielemans, S. Rathore, F. Engbrant, and P. Razifar, "Device-less gating for PET/CT using PCA," in Nuclear Science Symposium and Medical Imaging Conference (NSS/MIC). IEEE, 2011, pp. 3904-3910.

[3] O. Bertolli, S. Arridge, S. D. Wollenweber, C. W. Stearns, B. F. Hutton, and K. Thielemans, "Sign determination methods for the respiratory signal in data-driven pet gating," Physics in Medicine and Biology, vol. 62, no. 8, p. 3204, 2017.

[4] S. Vandenberghe, E. Mikhaylova, E. DHoe, P. Mollet, and J. Karp, "Recent developments in time-of-flight PET," EJNMMI physics, vol. 3, no. 1, p. 1, 2016.

[5] O. Bertolli, S. Arridge, C. W. Stearns, S. D. Wollenweber, B. F. Hutton, and K. Thielemans, "Data driven respiratory signal detection in PET taking advantage of time-of-flight data," in Nuclear Science Symposium and Medical Imaging Conference (NSS/MIC). IEEE, 2016, pp. 1-3.

[6] V. Bettinardi, L. Presotto, E. Rapisarda, M. Picchio, L. Gianolli, and M. Gilardi, "Physical performance of the new hybrid PET/CT Discovery690," Medical Physics, vol. 38, no. 10, pp. 5394-5411, 2011. 